\title{
A Proposal for Generating Good Assembly Sequences by Tournament Tree
}

\author{
Kenji Tsuboi $^{\dagger}$ \\ Manufacturing System Development BL, Business Promoting office \\ Honda Motor Co., Ltd., Tochigi, 329-1224, JAPAN \\ E-mail: kenji_tsuboi@hm.honda.co.jp \\ Toshiyuki Matsumoto \\ Department of Industrial and Systems Engineering \\ Aoyama Gakuin University, Kanagawa, 229-8558, JAPAN \\ E-mail: matsumoto@ise.aoyama.ac.jp \\ Shinji Shinoda \\ Department of Industrial Administration \\ Tokyo University of Science, Chiba, 278-8510, JAPAN \\ E-mail: shinoda@ia.noda.tus.ac.jp \\ Akira Niwa \\ Department of Electromechanics \\ Seikei University, Tokyo, 180-8633, JAPAN \\ E-mail: niwa@st.seikei.ac.jp
}

Received, February 9, 2010; Revised, September 29, 2010 / December 10, 2010 / February 16, 2011 ; Accepted, February 25, 2011

\begin{abstract}
In seeking further efficiency in production preparation, it is common to examine assembly sequences using digital manufacturing. The assembly sequences affect the product evaluation, so it is necessary to test several assembly sequences before actual production. However, because selection and testing of assembly sequences depends on the operator's personal experience and intuition, only a small number of assembly sequences are actually tested. Nevertheless, there is a systematic method for generating assembly sequences using a contact-related figure. However, the larger the number of parts, the larger the number of assembly sequences geometric becomes. The purpose of this study is to establish a systematic method of generating efficient assembly sequences regardless of the number of parts. To generate such assembly sequences selectively, a "Tournament Tree," which shows the structure of an assembly sequence, is formulated. Applying the method to assembly sequences of a water valve, good assembly sequences with the same structure as the Tournament Tree are identified. The structure of such a Tournament Tree tends to have fewer steps than the others. As a test, the structure is then applied for a drum cartridge with 38 parts. In all the assembly sequences generated from the contact-related figures, the best assembly sequence is generated by using the Tournament Tree.
\end{abstract}

Keywords: Digital Manufacturing, Assembly Sequence, Assembly Work, Tournament Tree

\section{INTRODUCTION}

Due to the deterioration in economic conditions and the rise in raw material prices, the life cycles of products have become shorter, and shortening the product lead time from development to mass production and reducing the cost have also become important issues in manufactur- ing. Considering such needs, a method called Virtual Factory is gathering increased attention because it has the potential to shorten the product lead time (Grieves, 2006; YIN Z-P, 2004; Chen et al., 2002; Molina, 1995). Virtual Factory conducts pre-production processes by simulating the assembly of products via 3D-CAD. Assembly sequences must be carefully designed, considering vari-

† : Corresponding Author 
ous alternatives, because they affect other factors such as the difficulty level of the assembly or the work layout. However, so far, only a limited number of assembly sequences are tested before production with a traditional method relying on a worker's experience and intuition. Although it is possible to create operation animations and show virtual operations conducted in a virtual factory by entering vast amounts of data manually using a motion capture apparatus or a computer mouse into DELMIA Corp. software DELMIA DPM ASSEMBLY and CATIA, this is not a realistic method for actual production because it is a time-consuming process.

There are many studies on assembly sequence planning (Jun et al., 2005; Martinez et al., 2009; Wen et al., 2008, 2010; Kai et al., 2008; Marian et al., 2006; Young et al., 2009; Wang et al., 2010; Qiang, 2009; Hongbo et al., 2009; Biswal et al., 2009; Zhou et al., 2008; Shanshan et al., 2008; Hwai et al., 2007; Ostrovsky et al., 2006; Liverani et al., 2006). In this research area, it is common to generate assembly sequences using a BOM method, a genetic algorithm method or a graph theory-based method. To generate assembly sequences with this method, using BOM while building a partly finished product unit, it is vital to clarify both the structure of the partly finished product and the precedence relations of the parts; otherwise, the assembly sequences are not finalized. To generate assembly sequences, a graph theorybased method requires both the structure of the partly finished product and the precedence relations of the parts. A genetic algorithm also requires the conduct of parts and the precedence relations of the parts to generate assembly sequences. Although they obtain and organize the information of a partly finished product unit, the precedence relations of the parts or the conduct of parts, those methods cannot generate assembly sequences. It takes such a long time that it is not of practical use. A method of generating assembly sequences with simple information is required.

Previously, a method of generating assembly sequences systematically using contact-related figures has been proposed (Shinoda et al., 2000, 2001, 2002,
2004, 2005a, 2005b, 2009; Niwa et al., 2009; Yamanaka et al., 2006; Tsuboi et al., 2006, 2007). In the studies, all the feasible assembly sequences are first generated using contact-related figures and then evaluated. However, as more parts or components are used in an assembly, such a method may need to deal with an exponentially growing number of assembly sequences, and therefore, it will be difficult to generate all the sequences and to evaluate the generated assembly sequences. For example, for product $\mathrm{A}$, which is made up of 42 parts, there are 5.12 million possible assembly sequences, despite the restrictions in the actual implementation of assembly operations. Because it may take a considerable amount of time to evaluate 5.12 million assembly sequences at the production preparation stage, this method has not been realistic for use at actual production sites.

This study thus aims to clarify the two objectives described below in order to generate efficient assembly sequences systematically and effectively out of many possible alternatives. The first aim is to devise a method of describing assembly sequences structurally and thus obtain a general idea of the characteristics of efficient assembly sequences. The second aim is to devise a method of selecting efficient assembly sequences by describing assembly sequences structurally. By achieving these two objectives, it is possible to select and evaluate only the efficient assembly sequences without generating and evaluating all the feasible assembly sequences.

\section{TOURNAMENT TREE: A NEW MODE OF EXPRESSION FOR GENERATING ASSEMBLY SEQUENCES}

The author has devised a new mode of expression for generating assembly sequences, called Tournament Tree (hereinafter denoted TT), by focusing on the fact that the same parts could be assembled in different orders and with different assembly sequences. Consider, for example, an "audio plug" that consists of 4 parts; it has been noted in a preceding study (Shinoda et al., 2004) that 3 assembly sequences can be generated, as illustrated

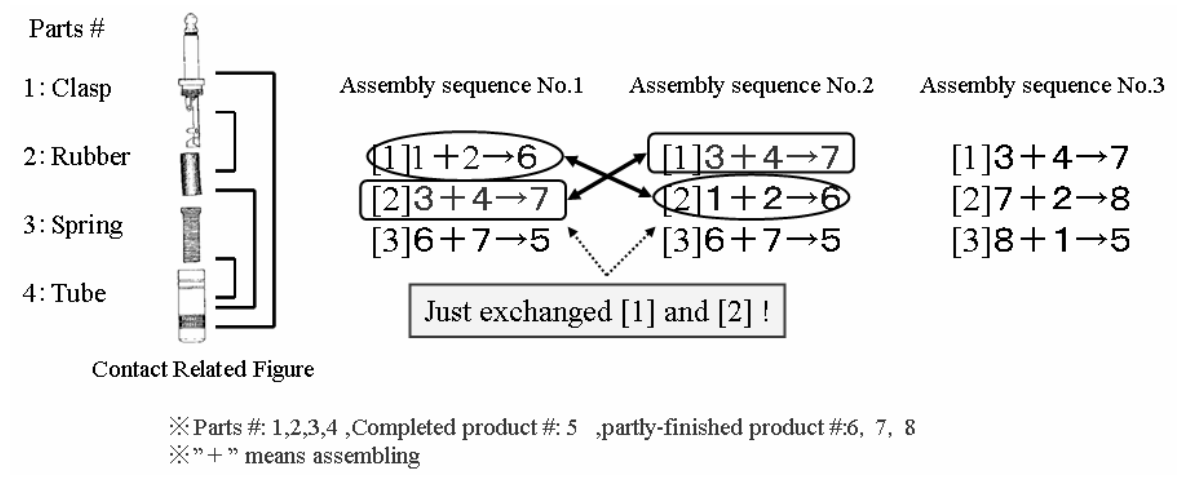

Figure 1. Audio Plug and 3 assembly sequences. 
in Figure 1. Taking a closer look at these assembly sequences, it is clear that the only difference between assembly sequence No.1 and 2 is the reversed order of assembly [1] and [2]. Also, it is clear that Assembly sequence No.3 has a totally different assembly order from Assembly sequences No.1 and 2. To clarify and express the difference between Assembly sequences No.1,2 and Assembly sequence No.3, the author has devised a new mode of expression with a new concept, TT.

TT represents the orders in which parts or components are assembled in a tournament style. It can describe assembly orders structurally, and thus, it can also express the precedence relations between parts visually. The procedure for producing TT from assembly sequences is as follows.

Step 1 : Lay out numbers matching parts numbers from left to right.

Step 2 : Connect the assembled parts with lines.

Step 3 : Draw lines between assembled partly finished products and single parts or partly finished products.

Step 4 : Repeat Steps 2 and 3 until no parts or partly finished products are left behind.

By following the above procedure, TT can be generated from assembly sequences. Figure 2 shows the TTs. produced for the assembly sequences presented in Figure 1. Assembly sequence No.1 and Assembly sequence No. 2 differ in the assembly order of [1] and [2], so they are expressed using the same TT. Assembly sequence No.3 has a different TT because the single parts are sequentially added to one partly finished product. TTs can clearly show the structural difference in assembly order between Assembly sequences No.1, 2 and Assembly sequence No.3. Thus, by expressing the assembly sequences with TTs, multiple assembly sequences with different assembly orders of parts can be integrated into one TT.

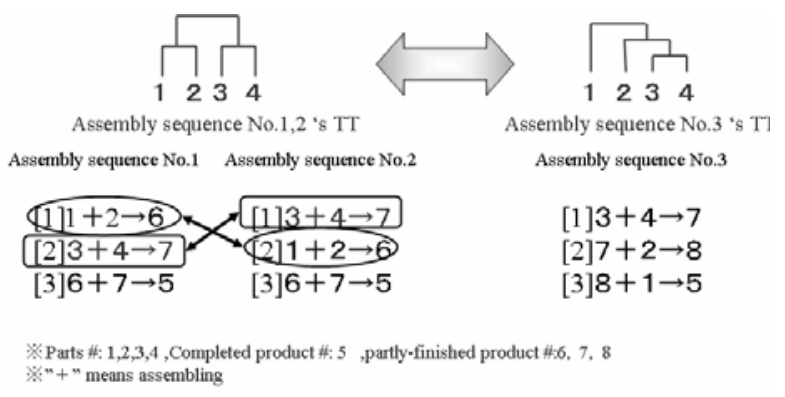

Figure 2. TTs of assembly sequence No.1, 2 and No.3.

\section{ANALYZING ASSEMBLY SEQUENCES USING TTS}

This study aims to generate efficient assembly sequences selectively out of numerous possible assembly sequences by considering the relationship between TTs and assembly sequences. However, it is not yet clear what TTs can generate efficient assembly sequences. Therefore, by listing all possible assembly sequences and producing TTs, the author aims to clarify the relationship between TTs and efficient assembly sequences. Here, a "water valve" illustrated in Figure 3 is taken as example of an assembly product. The water valve consists of 10 parts, and 1008 assembly sequences were specified and presented in a preceding study (Shinoda et al., 2004). TTs are produced for all these 1008 assembly sequences, and the relationship between TTs and efficient assembly sequences is clarified.

\subsection{Preliminary Conditions and Evaluation Items}

To conduct an assembly task following a corresponding assembly sequence, two preliminary conditions are given. The first condition is to conduct operations using both right and left hands. In an assembly task, a larger part is called a parent part, and a smaller part is called a child part, for convenience. When assembling parts using both hands, the left hand should hold the parent part, and the right hand should hold the child part. Upon the assembly of these parts, the child part held in the right hand should move toward the parent part in the left hand. The terms "parent part" and "child part" are identified by the relative sizes of the objects, and the terms differ from their meanings in the PLM research field (Ishii et al., 1992). The sizes of the parts can be obtained by 3D-CAD as in the preceding studies (Shinoda et al., 2000, 2001, 2002, 2004, 2005a, 2005b, 2009; Niwa et al., 2009; Yamanaka et al., 2006; Tsuboi et al., 2006, 2007). Table 1 shows the size of each part of the water valve.

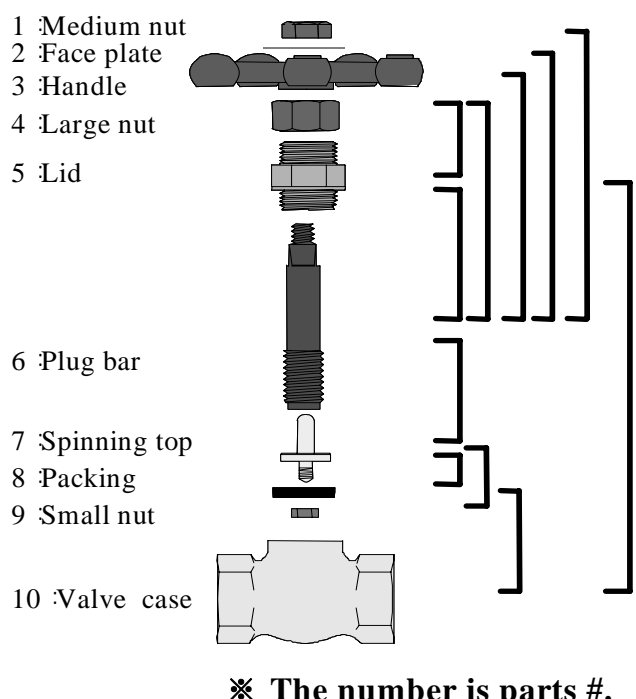

Figure 3. Water valve.

The second condition is to conduct and complete an 
assembly task top-to-bottom. This has been proved to be an easy order to perform an assembly task in a preceding study (Shinoda et al., 2004). Because by doing so, an operator can visually check the parts or areas of parent parts and child parts being attached. When assembling parts top-to-bottom, the child part held with the right hand is put together with the parent part in the left hand from above.

Efficient assembly sequences are judged by the evaluation items, including the number of steps and the total angle of rotation of each sequence. These evaluation items are specified when transferring assembly sequences into VFDL (Virtual Factory Description Language). VFDL is an instruction language for creating 3DCG of assembly works developed by Dr. Shinoda (Shinoda et al., 2004), and it describes any transformation included in an assembly as instructions. 3DCG can be generated by using VFDL and the software ENVISION released by DELMIA Corp. (FRN). Different assembly sequences have different numbers of steps and total angles of rotation. Based on the evaluation items, the efficiency of the assembly sequences can be judged.

One of the evaluation items discussed above, the number of steps, indicates the number of movements involved in an assembly. For example, moving the right hand to pick up a part, grasping a part, and assembling parts can all be counted as one step. The number of steps can roughly be converted to the time taken, and the fewer the steps, the shorter the time it takes to produce more products. Therefore, the TTs of assembly sequences that have fewer steps shall be described as efficient TTs.

Another evaluation item, the total angle of rotation, refers to the angle at which a part or partly finished product is rotated to complete an assembly top-to-bottom. Rotating a partly finished product also counts as one step in an assembly. However, there are cases when parts are not fixed to each other in the course of an assembly, and the resulting partly finished product is fragile. When such partly finished products are rotated, unfixed parts may fall off. Thus, TTs with less rotation should be selected as more efficient TTs.

Considering the two evaluation items above, efficient assembly sequences must have fewer steps and less rotation. The relationship between efficient assembly sequences and efficient TTs will be analyzed with each evaluation item below.

\subsection{Classifying TTs According to the Number of Assembly Sequences Generated}

The authors have succeeded in producing TTs for all 1008 possible assembly sequences of a water valve. Because there were overlaps, 334 TTs were produced, as shown in Figure 4. As characteristics, the same structure of TTs, which can reproduce the same number of assembly sequences, can be integrated into a single TT of the pattern by switching the numbers of the parts. For example, there are 189 TTs that can reproduce one assembly sequence, and thus, these TTs can be integrated into one TT of the pattern by replacing the numbers of parts. The TTs and their patterns are classified by the numbers of assembly sequences generated. The $334 \mathrm{TTs}$ mentioned earlier are classified into 13 patterns, from TT-A to TT-M, as shown in Figure 5. Figure 5 illustrates the patterns classified by the numbers of assembly sequences. For example, with TT-A, there are 189 TTs that can reproduce one assembly sequence, and with TT-M, there are 2 TTs that can reproduce 56 assembly sequences. The relationships between the patterns of TTs and efficient assembly sequences will be analyzed in the next section.

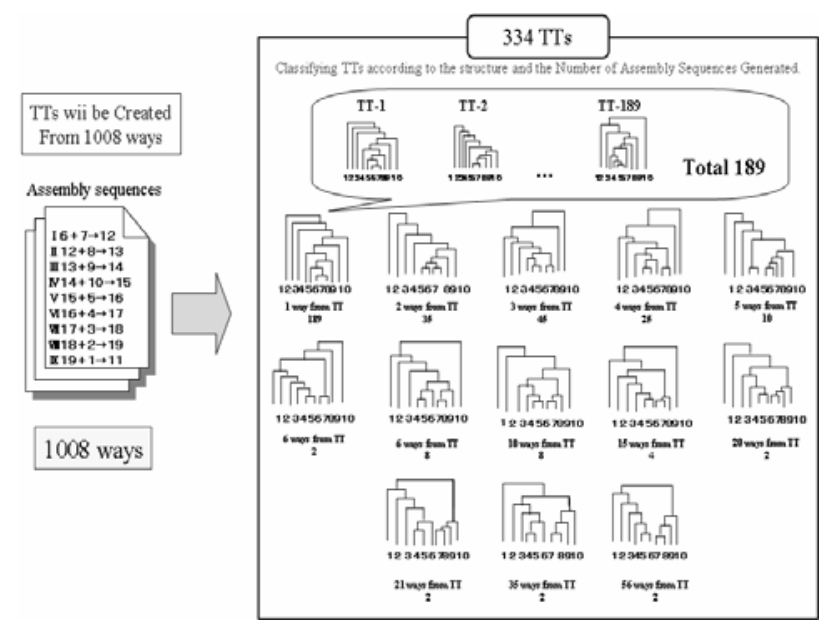

Figure 4. Water-valve's TTs.

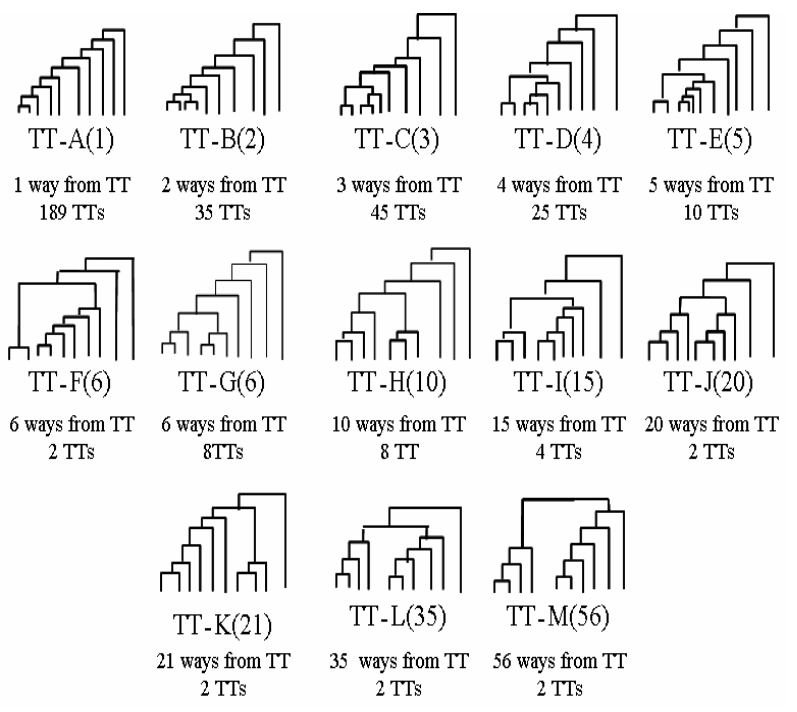

Figure 5. Classify the TTs according to the number of assembly sequences generated. 
Table 1. The sizes of the water valve parts.

unit: mm

\begin{tabular}{|c|c|c|c|}
\hline Parts No. & $\mathrm{X}$ & $\mathrm{Y}$ & $\mathrm{Z}$ \\
\hline \hline 1 & 11.34 & 9.82 & 4.95 \\
\hline 2 & 18.50 & 18.50 & 0.50 \\
\hline 3 & 52.12 & 52.09 & 9.51 \\
\hline 4 & 20.50 & 17.76 & 12.15 \\
\hline 5 & 21.00 & 21.00 & 24.10 \\
\hline 6 & 10.00 & 10.00 & 64.25 \\
\hline 7 & 15.00 & 15.00 & 25.70 \\
\hline 8 & 14.20 & 14.20 & 3.05 \\
\hline 9 & 6.08 & 5.28 & 2.60 \\
\hline 10 & 48.00 & 31.38 & 34.19 \\
\hline
\end{tabular}

\subsection{Analyzing the Number of Steps}

Figure 6 shows the distribution of the number of steps from TT-A to TT-M. TT-A has 65 to 76 steps on the distribution chart, while TT-M has 74 to 114 steps. Looking at the chart, we see that TTs that reproduce fewer assembly sequences generally have fewer steps, while TTs that reproduce more assembly sequences have more steps. As for the distribution, TTs with fewer assembly sequences are distributed unevenly, whereas TTs with more assembly sequences are distributed fairly widely.

With the TTs of fewer assembly sequences, the parts are consistently added to a single partly finished product; thus, there is less chance to use a temporary storage place, and the number of steps decreases. There- fore, TTs with TT-A patterns can be judged as efficient because the parts are consistently added to one parent part. However, the numbers of steps can vary within the assembly sequences of TT-A patterns. Differences may be caused by the arrangement of the parts; thus, the following section will discuss and analyze the arrangements of the parts in TT-A-type assembly sequences.

After analyzing how the arrangements of parts and the number of steps are related in TT-A, it is found that TTs that contain certain arrangements using a certain part have fewer steps. The part and arrangement in question are the ones using the part \# 6 plug bar as a connector for other connecting parts. Because the part \# 6 is the largest part used for the water valve, it is often used as a parent part in an assembly. If different parts perform the role of a parent part, an operator has to pick up and put down the parts held in the left hand, which increases the number of steps. Therefore, if a part often plays the role of a parent part, that part should be used first in an assembly, and the numbers of the parts should be arranged as in the TT, so as to generate assembly sequences with fewer steps. When the assembly sequences are shown with TTs, it is clearly more effective to assemble parts successively, without picking them up or putting them down so often.

\subsection{Analyzing the Total Angle of Rotation}

Figure 7 shows that the distribution of the total angle of rotation can be categorized into three kinds of angles: 900, 1260 and 1620 degrees. Comparing the distribution of the total angle of rotation with the TT patterns and with the number of steps, it is found that the distribution patters are different. The assembly sequence derived from the TT-A pattern has fewer steps

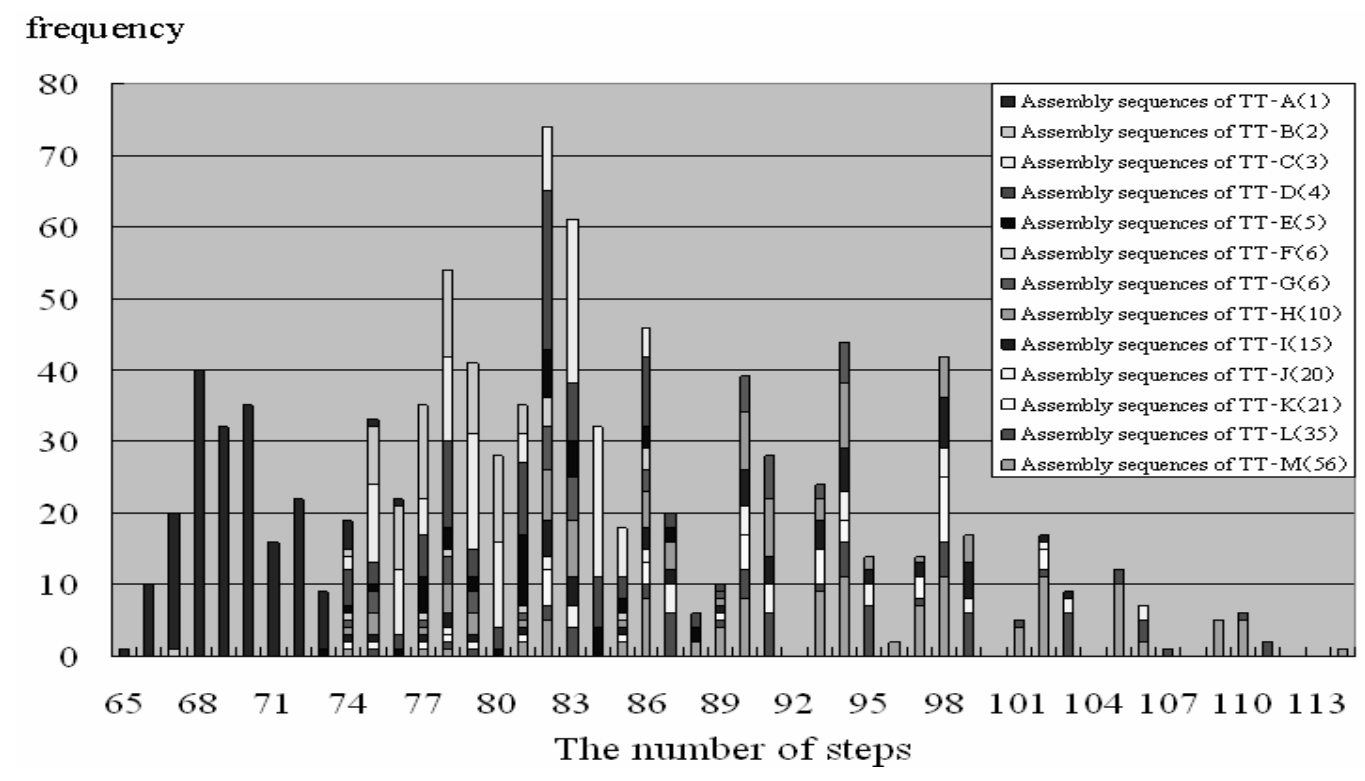

Figure 6. Distribution of steps in TT patterns 


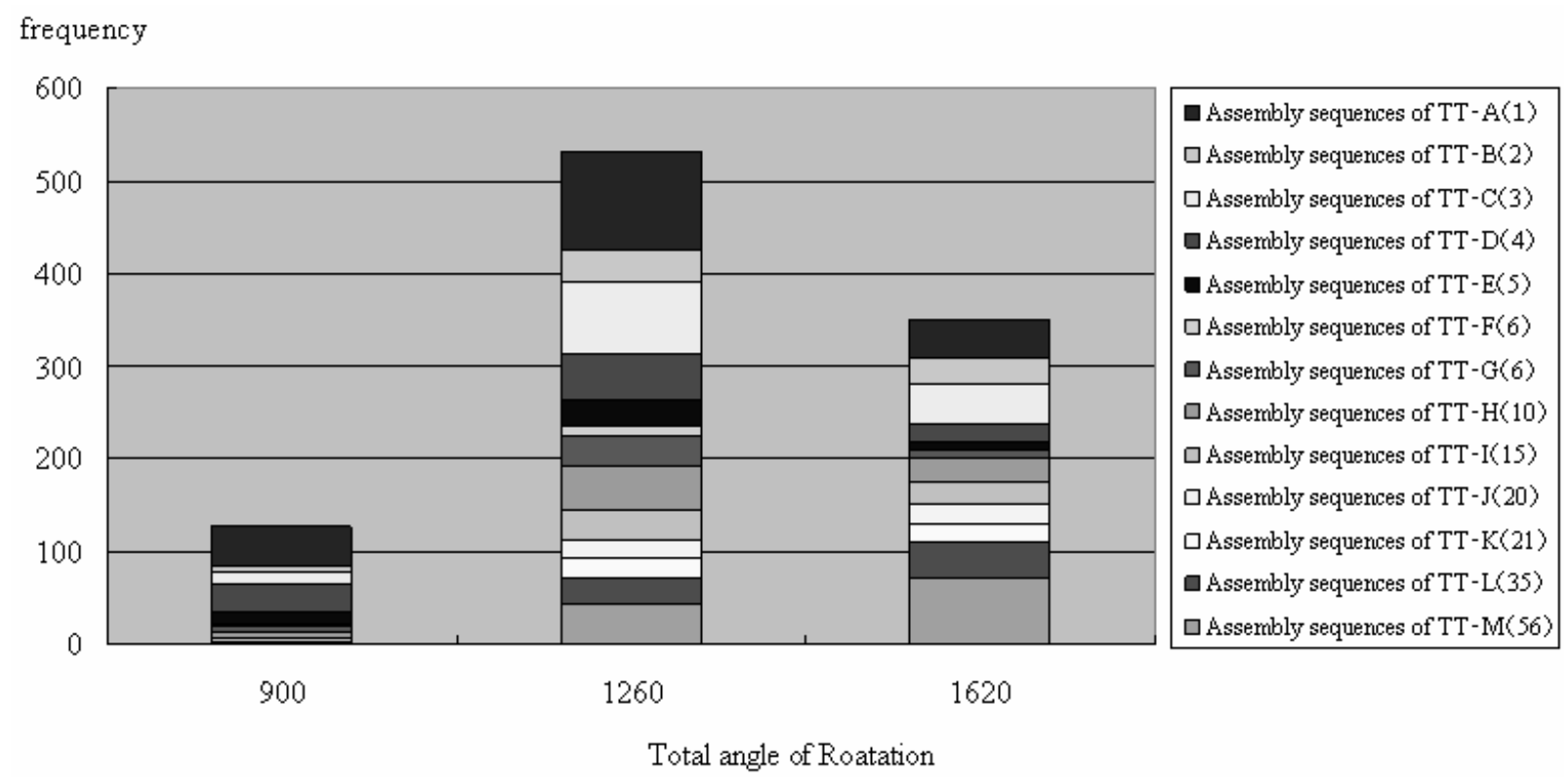

Figure 7. Distribution of total angle of rotation in TT patterns.

compared with other patterns, but the angle of rotation does not necessarily decrease as a function of the number of steps. It is thus possible to conclude that the TT patterns and angles of rotation are not related.

After considering which kinds of assembly sequences have less angle of rotation in an assembly, it was found that the assembly sequences have less total rotation when parts are continuously rotated and put together during an assembly. In the assembly of a water valve, the object of study here, parts must be rotated when parts \#6 and 7, 7 and 8, 7 and 9 are put together. By arranging the parts in a TT so that the assembly of relevant parts is conducted successively, it is possible to produce assembly sequences with less total rotation.

Analyzing the number of steps in 3.3, it becomes clear that when parts are put together successively with a single parent part, the relevant TTs have fewer steps and are higher in work efficiency. Analyzing 3.4, the total angle of rotation, it was found to be effective to order the numbers of parts connected to each branch of the TT so that the parts are assembled from one direction successively. Using the TTs, it is now possible to evaluate the assembly sequences with less rotation and fewer steps. In such assembly sequences, elaborate series of parts are successively added to a single parent part. Specifically, TTs can show the flaws in designing the assembly of a product, the problems in current assembly sequences and other possible problems. The next chapter discusses how to generate the efficient assembly sequences discussed above using TTs. It also deals with assembly sequence design using BOM, which focuses more on constructing partly finished product units. The difference between the two approaches lies in that the TTs put more emphasis on adding parts successively to a given parent part. In Chapter 4, the findings are applied to a product, and assembly sequences for an actual product are generated. The optimum assembly sequence will be identified by two evaluation items in Section 3.1.

\section{PROCEDURE OF SELECTIVELY GEN- ERATING ASSEMBLY SEQUENCES USING TTS}

In the preceding chapter, efficient TT patterns and arrangements of the numbers of parts are clarified. Based on these results, the procedure for generating efficient assembly sequences will be described below.

Step 1 : Select a parent part. The part with the largest number of contacts with other parts should be the parent part.

Step 2 : Produce a TT in which parts are put together with the parent part when possible.

Step 3 : Place the parent part at the initial assembly position.

Step 4 : Considering the precedence relations of the assembly order, when several parts can be assembled from one direction, arrange them in a row so that the assembly is done successively.

Step 5 : Considering the precedence relations of the assembly order, arrange the rest of the parts efficiently. 


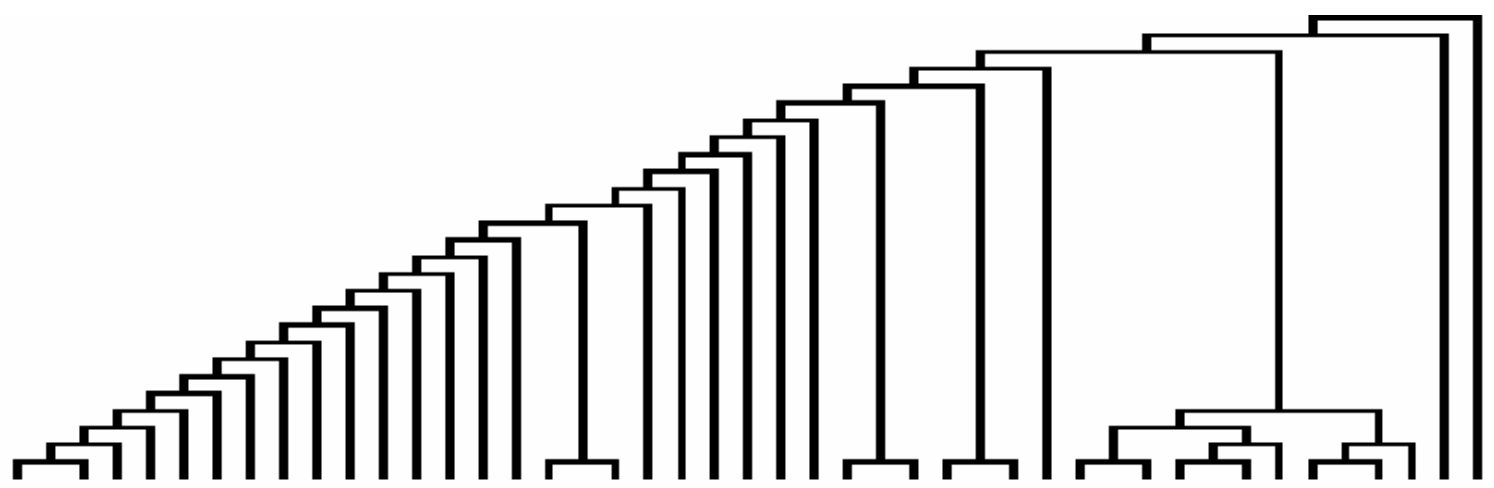

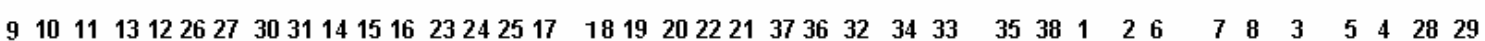

Figure 8. TT of the product B (drum cartridge).

By following the steps above, it is possible to generate efficient assembly sequences selectively from many alternative assembly sequences. To demonstrate and validate how effectively this method works, assembly sequences that include many steps will be used as an example. Product B, which is a "drum cartridge" that consists of 38 parts, is chosen as an object of study. 153664 assembly sequences are generated from the contactrelated figure (Shinoda et al., 2009). This drum cartridge is an actual product and is manufactured in a factory and distributed in the market. This product is different from product A, which consists of 42 parts. Figure 8 shows the TT generated by following the procedure of selectively generating efficient assembly sequences using TTs. The TT is designed so that parts are most likely to be put together with a parent part when possible in the assembly of the drum cartridge. Part \# 9 is most often used as a parent part, and thus, it is placed at the beginning of the assembly. Moreover, parts assembled from the same direction are arranged successively.

For the drum cartridge assembly, 72 types of assembly sequences are generated form the produced TTs. The assembly sequences and assembly directions are specified, and the number of steps and total angle of rotation are calculated from the transferred VFDL. The assembly sequences generated have between 295 and

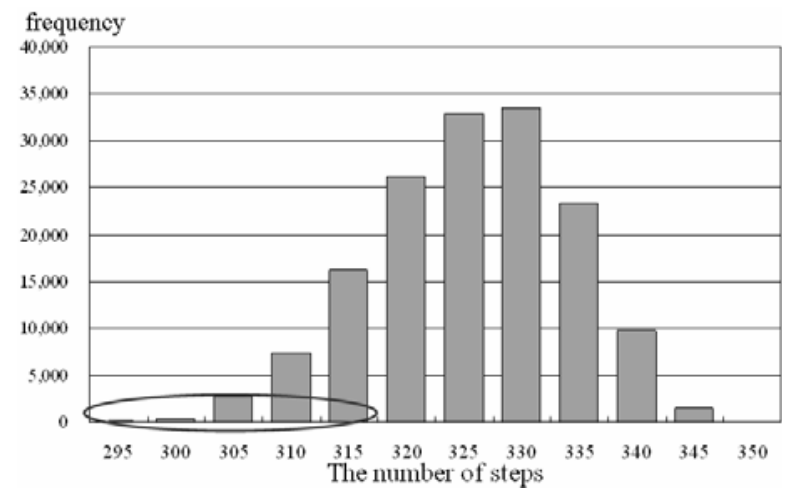

Figure 9. Distribution of product B steps.
315 steps, and the angles of rotation are either 3240 or 3250 degrees in total. Figure 9 shows that these assembly sequences are distributed at the point indicating the fewest steps on the chart and that they have the smallest angles of rotation. The result shows that using TTs, it is possible to generate only the efficient assembly sequences selectively. Here, the assembly sequence generated from the TTs and the actual assembly sequence used at the production site were compared. The actual assembly sequence had 305 steps, and it was clear that the TTgenerated sequence had fewer steps. If contact-related figures are to be used for generating assembly sequences, 153664 types of assembly sequences must be evaluated, whereas using TTs, the number of assembly sequences to be evaluated is limited to 72 types. Therefore, it is more efficient to use TTs for generating and evaluating assembly sequences. Therefore, it is clear that TTs can be used to generate only the efficient assembly sequences. The generating time of the TTs method and the contact-related figure method in the preceding study (Shinoda et al., 2004) is compared. The former takes one day and the latter takes 5 days; thus, the former is more efficient. Figure 10 shows 3DCG of the assembly sequence with the fewest steps. The operation is conducted using the TT-generated assembly sequence with

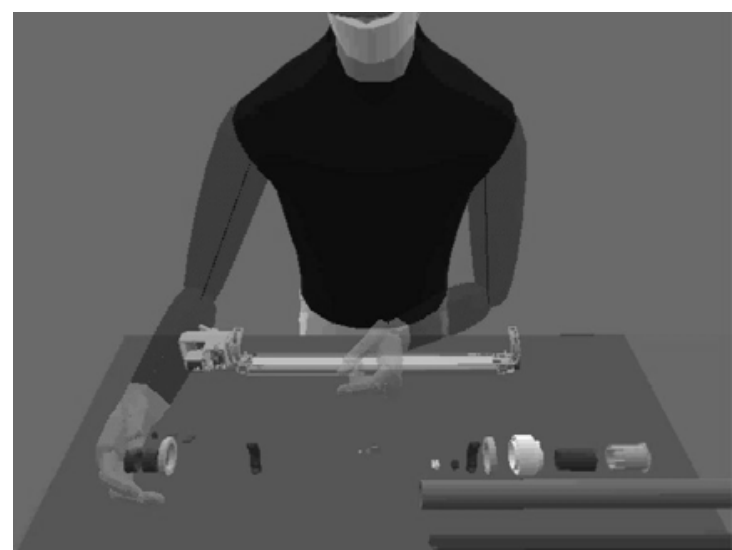

Figure 10. 3DCG of the assembly operation. 
the fewest assembly steps. This shows that it is possible to create efficient assembly sequences with fewer steps at the design stage using TTs.

It is thus possible to conclude that TTs can be used to generate assembly sequences and that TTs can be generated from assembly sequences. This means that TTs and assembly sequences are correlated. It is common to generate assembly sequences using BOM, a genetic algorithm-based method or a graph theory-based method. These methods cannot generate assembly sequences without the information of a partly finished product unit, the precedence relations of the parts or the conduct of the parts. However, when using TTs for generating assembly sequences, only the precedence relations need to be clarified before creating assembly sequences with minimal rotation of parts arranged successively. Compared with the method using the precedence relations of the parts only for generating assembly sequences (Jun et al., 2005; Martinez et al., 2009; Wen et al., 2008, 2010; Kai et al., 2008; Marian et al., 2006; Young et al., 2009; Wang et al., 2010; Qiang, 2009; Hongbo et al., 2009; Biswal et al., 2009; Zhou et al., 2008; Shanshan et al., 2008; Hwai et al., 2007; Ostrovsky et al., 2006; Liverani et al., 2006), the proposed method intentionally shows the assembly between two parts with a tournament style method, and the assembly sequences shown are visually accessible.

\section{CONCLUSION}

In this study, the author has devised and presented the idea of a Tournament Tree, which structurally expresses assembly sequences, in order to generate efficient assembly sequences systematically regardless of the number of parts used in an assembly. By clarifying the relationship between TTs and efficient assembly sequences, the effectiveness of a successive assembly was expressed in terms of TTs, and the author has succeeded in generating only the efficient assembly sequences selectively from TTs. Efficient assembly sequences can be generated regardless of the number of parts up to about 40, as shown in the application for Product B, which has 38 parts. Because it is possible to generate only the efficient assembly sequences by TTs, the concept of TTs may facilitate the implementation realization of effective pre-production methods in the future.

The number of steps and the total angle of rotation are used as indicators for determining efficient assembly sequences in this study. In an actual pre-production process, engineers also work to create exclusive jigs to promote work efficiency. In further research, the author plans to analyze the relationships between TTs and jigs and to develop a system that generates good assembly sequences using the concept of TT. By developing the concept of TT, it would be possible to construct not only effective assembly sequences but also effective disassembly sequences.

\section{REFERENCES}

Grieves, M. (2006), Digital Manufacturing in PLM Environments, CIM data, Michigan, U. S. A.

YIN, Z.-P. (2004), A virtual prototyping approach to generation and evaluation of mechanical assembly sequences, Proceedings of the Institution of $\mathrm{Me}$ chanical Engineers. Part B. Journal of Engineering Manufacture, 80-85.

Chen, C. L. P. and Yoh-Han, Pao (2002), An integration of neural network and rule-based systems for design and planning of mechanical assemblies, Systems, Man and Cybernetics, IEEE Transactions on, 23(5), 1359-1371.

Molina, A., Ellis T. I., Young R. I. M., and Bell R. (1995), Modeling Manufacturing Capability to Support Concurrent Engineering, Concurrent Engineering, 3(1), 29-42.

Jun, Du, Yuan-Yuan Jiao, and Jianxin Jiao (2005), Integrated BOM and routing generator for variety synchronization inassembly-to-order production, Journal of Manufacturing Technology Management, 16(2).

Martinez, M., Viet Pham, and Favrel, J. (2009), Optimal assembly plan generation: a simplifying approach, Journal of Intelligent Manufacturing, 20(1), 15-27.

Wen-Chin, Chen, Yung-Yuan, Hsu, Ling-Feng, Hsieh, and Pei-Hao, Tai (2010), A systematic optimization approach for assembly sequence planning using Taguchi method, DOE, and BPNN, Expert Systems with Applications, 37(1), 716-726.

Kai-Fu, Zhang, Hui, Cheng, and Yuan, Li (2008), Multi-objective harmonious colony-decision algorithm for more efficiently evaluating assembly sequences, Assembly Automation, 28(4), 348-355.

Marian, R. M., Luong, L. H. S., and Abhary, K. (2006), A genetic algorithm for the optimisation of assembly sequences, Computers and Industrial Engineering, 50(4), 503-527.

Young-Keun Choi, Dong Lee, and Yeong, Cho (2009), An approach to multi-criteria assembly sequence planning using genetic algorithms, International Journal of Advanced Manufacturing Technology, 42(1), 180-188.

Wang, Y. and Liu, J. H. (2010), Chaotic particle swarm optimization for assembly sequence planning, Robotics and Computer-Integrated Manufacturing, 26(2), 212-222.

Qiang, Su (2009), A hierarchical approach on assembly sequence planning and optimal sequences analyzing, Robotics and Computer-Integrated Manufacturing, 25(1), 224-234. 
Hongbo, Shan, Shenhua, Zhou, and Zhihong, Sun (2009), Research on assembly sequence planning based on genetic simulated annealing algorithm and ant colony optimization algorithm, Assembly Automation, 29(3), 249-256.

Biswal, B. B., Sharma, S., and Dash, P. (2009), Correct assembly sequence for robotic assembly using motion instability and part contact-level graphs, International Journal of Computer Applications in Technology, 36(2), 149-158.

Zhou, Xiaoming and Du, Pingan (2008), A model-based approach to assembly sequence planning, International Journal of Advanced Manufacturing Technology, 39(9), 983-994.

Wen-Chin, Chen, Pei-Hao Tai, Wei-Jaw, Deng, and Ling-Feng, Hsieh (2008), A three-stage integrated approach for assembly sequence planning using neural networks, Expert Systems with Applications, 34(3), 1777-1786.

Shanshan, Zhao and Zongbin, Li (2008), A new assembly sequences generation of three dimensional product based on polychromatic sets, Information Technology Journal, 7(1), 112-118.

Hwai-En, Tseng, Wen-Pai, Wang, and Hsun-Yi, Shih (2007), Using memetic algorithms with guided local search to solve assembly sequence planning, Expert Systems with Applications, 33(2), 451-467.

Qiang, Su (2007), Computer aided geometric feasible assembly sequence planning and optimizing, International Journal of Advanced Manufacturing Technology, 33(1-2), 48-57.

Ostrovsky-Berman, Y. and Joskowicz, L. (2006), Relative position computation for assembly planning with planar toleranced parts, International Journal of Robotics Research, 25(2), 147-170.

Liverani, A., Amati, G., and Caligiana, G. (2006), Interactive control of manufacturing assemblies with mixed reality, Integrated Computer-Aided Engineering, 13(2), 163-172.

Shinoda, S. and Niwa, A. (2000), A Fundamental Study of Listing Alternative Ideas for Designing a Work Process-A Case of Assembly-type Work- (in Japanese), Journal of Japan Industrial Management Association, 51, 321-329.

Shinoda, S. and Niwa, A. (2001), The Method of Representing Assembly-type Work by a Series of State/ Change Transition Diagrams in a Matrix Form (in Japanese), Journal of Japan Industrial Management Association, 52, 60-67.

Shinoda, S. and Niwa, A. (2002), A Fundamental Study of Constructing a System of Listing Alternative Methods for Designing Assembly-type Work (in
Japanese), Journal of Japan Industrial Management Association, 53, 127-138.

Shinoda, S. and Niwa, A. (2004), Method for Analysis of Attaching Relationship and Generation of All Assembly Sequence in Assembly Parts.-In the case of assembly parts which have single axis structure(in Japanese), IE Review, 45, 80-85.

Shinoda, S. and Niwa, A. (2005a), A Fundamental Study of Method in Designing 3-Dimentional Computer Graphics of Assembly-Type Works (in Japanese), Proceedings of 18th International Conference on Production Research, (CD-R).

Shinoda, S. and Niwa, A. (2005b), A Proposal of a Method Utilizing 3-DCG for Assembly-Type Works from the Viewpoint of an Essential Transformation, Journal of The Society of Plant Engineers Japan, 17(2), 91-97.

Shinoda, S., Shimozawa, K., Niwa, A., Kawase, K., Matsumoto, T., and Mizumachi, T. (2009), A Proposal for Prototype-free Production Preparation Processes Utilizing 3DCG Animations, The Journal of Industrial Engineering and Management Systems, 8(2), 109-120.

Niwa, A., Shinoda, S., and Kawase, K. (2009), A Basic Research on Methodology for Verifying Properties of All the Partly-finished Products and the Assembly Tasks before the Final Generation of All the Assembly Sequences-For Single-axis Structure Product Assembly-, Journal of The Society of Plant Engineers Japan, 20(3), 57-64, (in Japanese).

Yamanaka, H., Matsumoto, T., Shinoda, S., and Niwa, A. (2006), A Basic study for creating 3DCG animation of an assembly work, Proceedings of the 11th International Conference on Industrial Engineering Theory, Applications, and Practice, 913918.

Tsuboi, K., Matsumoto, T., Niwa, A., Shinoda, S., and Shimozawa, K. (2006), A Study of Evaluating the Assembly Sequence by Tournament Tree-A study for developing the Prototypeless Production System (2nd Report), Proceedings of the 17th Fall Conference on Japan Industrial Management Association, 4-5 (in Japanese).

Tsuboi, K., Matsumoto, T., Shinoda, S., and Niwa, A. (2007), A Basic Study for Producing Good Assembly Sequences by the Tournament Tree, Proceedings of the 8th Asia Pacific Industrial Engineering and Management System and 2007 Chinese Institute of Industrial Engineering Conference, Paper ID. 179.

Ishii, K. and Ichimura, T. (1992), A process design approach based on the fusion model, Technovation, 12(7). 